PROCEEDINGS OF THE

AMERICAN MATHEMATICAL SOCIETY

Volume 126, Number 7, July 1998, Pages 2071-2075

S 0002-9939(98)04574-

\title{
INTEGRAL MEANS AND PÓLYA FACTORIZATIONS
}

\author{
URI ELIAS
}

(Communicated by Hal L. Smith)

\begin{abstract}
Integral means of functions and their derivatives are studied. We find a relation between integral means and the Pólya factorization of ordinary linear differential operators.
\end{abstract}

The purpose of this note is to present relations between integral means of functions and the corresponding integral means of their derivatives. Also, we point out that these relations are consequences of the Pólya factorization of differential operators.

We begin with two identities:

Theorem 1. Let there be given the integral mean with weight function $w_{0}>0$,

$$
F(t)=\frac{\int_{a}^{t} w_{0}(s) f(s) d s}{\int_{a}^{t} w_{0}(s) d s} .
$$

(a) If $w_{1}=\int_{a}^{t} w_{0}$, then the generalized $k$-th derivatives $L_{k} f=\left(\frac{w_{1}}{w_{0}} \frac{d}{d t}\right)^{k} f$ have the integral means

$$
L_{k} F(t)=\frac{\int_{a}^{t} w_{0}(s) L_{k} f(s) d s}{\int_{a}^{t} w_{0}(s) d s}, \quad k=1,2, \ldots .
$$

(b) Let $w_{k}=\int_{a}^{t} w_{k-1}, \quad r_{k}=w_{k}^{2} / w_{k-1} w_{k+1}, \quad k=1,2, \ldots$. Then the integral mean with weight $w_{k}$ satisfies

$$
r_{k} \frac{d}{d t} r_{k-1} \frac{d}{d t} \ldots r_{1} \frac{d}{d t} F(t)=\frac{\int_{a}^{t} w_{k}(s) f^{(k)}(s) d s}{\int_{a}^{t} w_{k}(s) d s}, \quad k=1,2, \ldots
$$

Proof. By (1), $F(t)=\int_{a}^{t} w_{0}(s) f(s) d s / w_{1}(t)$, with $w_{1}(t)=\int_{a}^{t} w_{0}$. By differentiation and then integration by parts (with $w_{1}(a)=0$ ),

$$
\begin{aligned}
F^{\prime} & =w_{1}^{-2}\left[w_{0} f w_{1}-w_{0} \int_{a}^{t} w_{0} f\right] \\
& =w_{1}^{-2}\left[w_{0} f w_{1}-w_{0}\left(w_{1} f-\int_{a}^{t} w_{1} f^{\prime}\right)\right] \\
& =w_{1}^{-2} w_{0} \int_{a}^{t} w_{1} f^{\prime} .
\end{aligned}
$$

Received by the editors January 1, 1997.

1991 Mathematics Subject Classification. Primary 26A06, 26D10, 34A30.

Key words and phrases. Integral means, Pólya factorization.

(C) 1998 American Mathematical Society 
This may be written as

$$
\frac{w_{1}}{w_{0}} F^{\prime}=\frac{1}{w_{1}} \int_{a}^{t} w_{1} f^{\prime}=\frac{1}{w_{1}} \int_{a}^{t} w_{0}\left(\frac{w_{1}}{w_{0}} f^{\prime}\right)
$$

which is precisely (2) with $k=1, L_{1}=\frac{w_{1}}{w_{0}} \frac{d}{d t}$. Induction on $k$ completes the proof of (2).

Let us multiply (4) by $w_{1} / w_{2}$, where $w_{2}=\int_{a}^{t} w_{1}$, and rewrite it as

$$
\frac{w_{1}^{2}}{w_{0} w_{2}} F^{\prime}(t)=\frac{\int_{a}^{t} w_{1}(s) f^{\prime}(s) d s}{\int_{a}^{t} w_{1}(s) d s} .
$$

When this argument is repeated $k$ times with $w_{\ell+1}=\int_{0}^{t} w_{\ell}, \ell=1,2, \ldots, k,(3)$ follows.

Take for example the weight function $w_{0}(t)=t^{p}, p>-1$, and $a=0$. Then one has $w_{k}=t^{p+k} /(p+1) \ldots(p+k), w_{1} / w_{0}=t /(p+1), r_{k}=w_{k}^{2} / w_{k-1} w_{k+1}=$ $(p+k+1) /(p+k)$. If

$$
F(t)=\frac{\int_{0}^{t} s^{p} f(s) d s}{\int_{0}^{t} s^{p} d s}
$$

then (2) becomes

$$
\left(t \frac{d}{d t}\right)^{k} F(t)=\frac{\int_{0}^{t} s^{p}\left(s \frac{d}{d s}\right)^{k} f(s) d s}{\int_{0}^{t} s^{p} d s}, \quad k=1,2, \ldots,
$$

and (3) becomes

$$
\frac{p+k+1}{p+1} F^{(k)}(t)=\frac{\int_{0}^{t} s^{p+k} f^{(k)}(s) d s}{\int_{0}^{t} s^{p+k} d s}, \quad k=1,2, \ldots .
$$

(7) yields easily

$$
t^{k} F^{(k)}(t)=\frac{\int_{0}^{t} s^{p+k} f^{(k)}(s) d s}{\int_{0}^{t} s^{p} d s}, \quad k=1,2, \ldots .
$$

Since $\left(t \frac{d}{d t}\right)^{n}=t^{n} \frac{d^{n}}{d t^{n}}+\sum_{1}^{n-1} c_{i} t^{i} \frac{d^{i}}{d t^{i}}$ with certain constants $c_{i}$, one can deduce (8) also from (6).

Many other identities about integral means are available. For example, if we transform (5) and (6) by $s=u^{-1}, s \frac{d}{d s}=-u \frac{d}{d u}$, we get that for $q>1, v>0$,

$$
F(v)=\frac{\int_{v}^{\infty} u^{-q} f(u) d u}{\int_{v}^{\infty} u^{-q} d u} \quad \text { implies } \quad\left(v \frac{d}{d v}\right)^{k} F(v)=\frac{\int_{v}^{\infty} u^{-q}\left(u \frac{d}{d u}\right)^{k} f(u) d u}{\int_{v}^{\infty} u^{-q} d u},
$$

$k=1,2, \ldots$ If we choose $a=\infty, w_{0}=e^{-p t}, w_{k}=\int_{t}^{\infty} w_{k-1}=e^{-p t} / p^{k}$, then by (3), $r_{k}=1$ and

$$
F(t)=\frac{\int_{t}^{\infty} e^{-p s} f(s) d s}{\int_{t}^{\infty} e^{-p s} d s} \quad \text { implies } \quad F^{(k)}(t)=\frac{\int_{t}^{\infty} e^{-p s} f^{(k)}(s) d s}{\int_{t}^{\infty} e^{-p s} d s} .
$$


Corollary 1. Let $w_{0}=t^{p}$ as in (5).

(a) If $f^{(k)}$ has a fixed sign for $t \geq 0$, then $F^{(k)}$ has the same fixed sign as well.

(b) If $f, f^{\prime}, \ldots f^{(\ell)} \geq 0$ for $t \geq 0$, then

$$
F^{(k)}(t) \leq \frac{p+1}{p+k+1} f^{(k)}(t), \quad t \geq 0, \quad k=0, \ldots, \ell-1 .
$$

(c) If $f, f^{\prime}, \ldots f^{(\ell)} \geq 0, f^{(\ell+1)} \leq 0$, then

$$
0 \leq \frac{p+1}{p+\ell+1} f^{(k)}(t) \leq F^{(k)}(t), \quad t \geq 0, \quad k=0, \ldots, \ell .
$$

Proof. (a) is a consequence of (7). By (7) also

$$
\begin{aligned}
\frac{t^{p+k+1}}{p+1} F^{(k)}(t) & =\int_{0}^{t} s^{p+k} f^{(k)}(s) d s \\
& =\frac{t^{p+k+1}}{p+k+1} f^{(k)}(t)-\int_{0}^{t} \frac{s^{p+k+1}}{p+k+1} f^{(k+1)}(s) d s,
\end{aligned}
$$

and the upper bound (9) follows for $k+1 \leq \ell$.

If $f^{(\ell+1)} \leq 0$, then by (11) (with $k=\ell$ )

$$
F^{(\ell)}(t) \geq \frac{p+1}{p+\ell+1} f^{(\ell)}(t), \quad t \geq 0,
$$

which is (10) for $k=\ell$. Integration yields

$$
F^{(\ell-1)}(t)-\frac{p+1}{p+\ell+1} f^{(\ell-1)}(t) \geq F^{(\ell-1)}(0)-\frac{p+1}{p+\ell+1} f^{(\ell-1)}(0), \quad t \geq 0 .
$$

But by $(7), F^{(k)}(0)=((p+1) /(p+k+1)) f^{(k)}(0)$ for $k=1,2, \ldots$ Using this with $k=\ell-1,(12)$ becomes

$$
F^{(\ell-1)}(t)-\frac{p+1}{p+\ell+1} f^{(\ell-1)}(t) \geq\left(\frac{p+1}{p+\ell}-\frac{p+1}{p+\ell+1}\right) f^{(\ell-1)}(0) \geq 0
$$

for $t \geq 0$. Thus we get the lower bound (10) for $k=\ell-1$. Repeated integrations of the last inequality prove (10) for $k=\ell-2, \ldots, 1,0$.

Corollary 1 shows how convexity properties are inherited by averaging. About the convexity of $F(t)=t^{-1} \int_{0}^{t} f(s) d s$, see [Mit, §1.4.7] and references there. Part (a) of Corollary 1 also implies, for example,

Corollary 2. Let $f$ be completely monotone, that is, $(-1)^{n} f^{(n)} \geq 0, n=0,1, \ldots$. Then the average $F$ in (5) is completely monotone, too. If $f$ is completely convex, i.e. $(-1)^{n} f^{(2 n)} \geq 0, n=0,1, \ldots$, then $F$ of $(5)$ is also completely convex.

The integral identities (2),(3) may also be written as differential identities. For example, (1) and (2) are equivalent, respectively, to

$$
\frac{d}{d t}\left(w_{1} F\right)=w_{0} f \quad \text { and } \quad \frac{d}{d t}\left(w_{1} L_{k} F\right)=w_{0} L_{k} f .
$$

If we substitute $f=w_{0}^{-1} \frac{d}{d t}\left(w_{1} F\right)$ from the first equation into the second one, we get

$$
\frac{d}{d t}\left(w_{1} L_{k} F\right)=w_{0} L_{k}\left(w_{0}^{-1} \frac{d}{d t} w_{1} F\right)
$$


However, $L_{k}$ is the differential operator $\left(\frac{w_{1}}{w_{0}} \frac{d}{d t}\right)^{k}$, so each side of the last equation may be presented as a product of differential operators

$$
\frac{1}{w_{0}} \frac{d}{d t}\left(w_{1}\left(\frac{w_{1}}{w_{0}} \frac{d}{d t}\right)^{k} F\right) \equiv\left(\frac{w_{1}}{w_{0}} \frac{d}{d t}\right)^{k}\left(\frac{1}{w_{0}} \frac{d}{d t} w_{1} F\right) .
$$

Similarly, (1) and (3) are equivalent, respectively, to

$$
\frac{d}{d t}\left(w_{1} F\right)=w_{0} f \quad \text { and } \quad \frac{d}{d t} w_{k+1} \frac{w_{k}^{2}}{w_{k-1} w_{k+1}} \frac{d}{d t} \ldots \frac{w_{1}^{2}}{w_{0} w_{2}} \frac{d}{d t} F=w_{k} \frac{d^{k}}{d t^{k}} f
$$

which imply, as above, the differential operator identity

$$
\frac{1}{w_{k}} \frac{d}{d t} \frac{w_{k}^{2}}{w_{k-1}} \frac{d}{d t} \frac{w_{k-1}^{2}}{w_{k-2} w_{k}} \ldots \frac{w_{1}^{2}}{w_{0} w_{2}} \frac{d}{d t} F \equiv \frac{d^{k}}{d t^{k}}\left(\frac{1}{w_{0}} \frac{d}{d t} w_{1} F\right), \quad w_{i}=\int w_{i-1} .
$$

(13) represents two different Pólya factorizations of the same differential operator, say

$$
\alpha_{k+1} \frac{d}{d t} \alpha_{k} \ldots \alpha_{1} \frac{d}{d t} \alpha_{0} F \equiv \beta_{k+1} \frac{d}{d t} \beta_{k} \ldots \beta_{1} \frac{d}{d t} \beta_{0} F, \quad \alpha_{i}, \beta_{i}>0 .
$$

The same holds for (14). For more details about such factorizations, see [Pol], [Tre], [Cop]. One way to verify such identities is to show that the $(k+1)$ st order linear differential operators on both sides have the same leading coefficient $\alpha_{k+1} \ldots \alpha_{0}=$ $\beta_{k+1} \ldots \beta_{0}$ and the same null space. For example, for (13) it follows by direct calculation (and using repeatedly $w_{0}=w_{1}^{\prime}$ ) that the null spaces of both sides are spanned by the $k+1$ functions $\left\{w_{1}^{-1}, 1, \log w_{1},\left(\log w_{1}\right)^{2}, \ldots,\left(\log w_{1}\right)^{k-1}\right\}$ and $\alpha_{k+1} \ldots \alpha_{0}=\beta_{k+1} \ldots \beta_{0}=\left(w_{1} / w_{0}\right)^{k+1}$.

Now we turn to the inverse question, namely are any two factorizations like (15) of a differential operator a source of integral mean identities like (2) and (3)? If (15) is factored into products of differential operators

$$
\frac{1}{v_{0}} \frac{d}{d t}\left(v_{1} N_{k}[F]\right) \equiv M_{k}\left(\frac{1}{w_{0}} \frac{d}{d t}\left(w_{1}[F]\right)\right),
$$

where $M_{k}, N_{k}$ are $k$ th order differential operators and $v_{0}^{-1} \frac{d}{d t} v_{1}, w_{0}^{-1} \frac{d}{d t} w_{1}$ are first order differential operators, $v_{1}=\int v_{0}, w_{1}=\int w_{0}$, then

$$
F(t)=\frac{\int w_{0} f}{\int w_{0}} \quad \text { implies } \quad N_{k}[F](t)=\frac{\int v_{0} M_{k}[f]}{\int v_{0}} .
$$

(15) can be always written as (16) in a trivial way. All one has to do is to multiply (15) from the right and the left hand sides by suitable factors and split $\alpha_{k}, \beta_{1}$ into products. This is, of course, useless, since multiplying, say, $v_{0}$ by a positive function leads to multiplication of $M_{k}$ by the reciprocal of the same function. So, the real question is to obtain interesting factorizations (16) with "nice" operators $M_{k}, N_{k}$. We exhibit such a result, based on the identity

$$
t^{-n+1}\left(\frac{d}{d t}\right) t^{n}\left(\frac{d}{d t}\right)^{2 n-1} F \equiv\left(\frac{d}{d t}\right)^{2 n-1} t^{n}\left(\frac{d}{d t}\right) t^{-n+1} F
$$

(17) is easily established, since both sides are equal to $t F^{(2 n)}+n F^{(2 n-1)}$. 
Theorem 2. Let $f \in C^{2 n-1}[0, \infty), t^{n} f \in L\left(t_{0}, \infty\right)$ and $f^{(n-1)}(0)=0$. If

$$
F(t)=\frac{\int_{t}^{\infty} s^{-n} f(s) d s}{\int_{t}^{\infty} s^{-n} d s}, \quad 0 \leq t<\infty
$$

then

$$
\frac{n}{n-1} F^{(2 n-1)}(t)=\frac{\int_{0}^{t} s^{n-1} f^{(2 n-1)}(s) d s}{\int_{0}^{t} s^{n-1} d s} .
$$

Proof. Let $F(t)$ be defined by (18), that is, $t^{-n+1} F(t) /(n-1)=\int_{t}^{\infty} s^{-n} f(s) d s$, or

$$
(n-1) f(t)=t^{n} \frac{d}{d t}\left(t^{-n+1} F(t)\right) .
$$

We differentiate (20) $2 n-1$ times and get by the identity (17) that

or

$$
(n-1) f^{(2 n-1)}=\frac{d^{2 n-1}}{d t^{2 n-1}} t^{n} \frac{d}{d t}\left(t^{-n+1} F\right)=t^{-n+1} \frac{d}{d t}\left(t^{n} F^{(2 n-1)}\right),
$$

$$
\left.t^{n} F^{(2 n-1)}\right|_{0} ^{t}=(n-1) \int_{0}^{t} s^{n-1} f^{(2 n-1)} d s .
$$

The lower limit has to be calculated carefully due to the definition of $F$ at $t=0$. To determine the behaviour of $F$ and its derivatives near $t=0$, we differentiate (20) $n-1$ times,

$$
(n-1) f^{(n-1)}=\left(t^{n}\left(t^{-n+1} F\right)^{\prime}\right)^{(n-1)}=\left(t F^{\prime}+(-n+1) F\right)^{(n-1)}=t F^{(n)},
$$

i.e., $F^{(n)}=(n-1) f^{(n-1)}(t) t^{-1}$. By $n-1$ more differentiations and passing to limit as $t \rightarrow 0$, it follows that $\left.t^{n} F^{(2 n-1)}\right|_{0}=(-1)^{n}(n-1)(n-1) ! f^{(n-1)}(0)$, and this equals 0 due to our assumption. Thus (21) becomes

$$
t^{n} F^{(2 n-1)}(t)=(n-1) \int_{0}^{t} s^{n-1} f^{(2 n-1)} d s,
$$

which is precisely (19).

Beginning with the identity

$$
t^{-n+1} \frac{d}{d t} t^{n+1}\left(\frac{d}{d t}\right)^{n} \equiv\left(\frac{d}{d t}\right)^{n} t^{n+1} \frac{d}{d t} t^{-n+1},
$$

it can be shown that (18) implies

$$
\frac{n}{n-1} t F^{(n)}(t)=\frac{\int_{0}^{t} s^{n-1}(s f(s))^{(n)} d s}{\int_{0}^{t} s^{n-1} d s} .
$$

\section{REFERENCES}

[Cop] Coppel, W. A., Disconjugacy, Lecture Notes in Math., vol. 200, Springer Verlag, Berlin, 1971. MR 57:778

[Mit] Mitrinovic, D. S., Analytic inequalities, Springer Verlag, Berlin, 1970. MR 43:448

[Pol] Pólya, G., On the mean value theorem corresponding to a given linear homogeneous differential equation, Trans. Amer. Math. Soc. 24 (1924), 312-324.

[Tre] Trench, W. F., Canonical forms and principal systems for general disconjugate equations, Trans. Amer. Math. Soc. 189 (1974), 319-327. MR 48:8969

Department of Mathematics, Technion, Haifa 32000, Israel

E-mail address: elias@techunix.technion.ac.il 